\title{
MetroNet: A Metropolitan Simulation Model Based on Commuting Processes
}

\author{
Efrat Blumenfeld-Lieberthal ${ }^{1}$ and Juval Portugali ${ }^{2}$ \\ ${ }^{1}$ The Azrieli School of Architecture, Tel Aviv University, Israel \\ efratbl@post.tau.ac.il \\ ${ }^{2}$ The Department of Geography and Human Environment, Tel Aviv University, Israel \\ juvalepost.tau.ac.il
}

\begin{abstract}
The aim of this work is to identify a set of fundamental rules that govern the interactions within urban systems at the metropolitan scale. For that, we developed an USM (Urban Simulation Model) specifically designed to study the evolution and dynamics of systems of cities. Our model is innovative in its structure: it is a superposition of cellular automata and agent based modeling approaches (that are essentially spatial analyses) and a complex network approach (that is essentially a topological analysis). This implies that in our model, the local activities and interaction of agents give rise to the global urban structure and network that in turn affects the agents' cognition, behavior, movement and action in the city and so on in circular causality. The model simulates commuting patterns of agents within a metropolis. The agents in our model represent workers who look for working places, the nodes represent urban employment centers, and the links represent commuters. Our results address three issues: the first suggests that the perception of urban boundaries plays a significant role in the metropolitan evolution in terms of network topology. This means that the existence of business centers, located in proximity to each other (but belonging to different municipalities) may lead to the emergence of new centers at the metropolis scale. The second issue concerns urban segregation; our results suggest that the location preferences of the agents regarding proximity to similar/different agents have a major affect not only on the urban morphology but also on the topology of the urban network. The third and last issue concerns the size distributions of agents in our model; these distributions correspond to all types of homogenous distributions observed in real system of cities.
\end{abstract}

Keywords: urban complexity, urban networks, agent based models, urban simulation models.

\section{Introduction}

In 1965, Christopher Alexander in his paper "A city is not a tree" described the differences between the way modernist planners grasp the relationships between urban entities and the way they exist in reality. A careful reading of this work suggests that Alexander was one of the first scholars that proposed a new understanding of cities as 
complex systems that can be described as complex networks. These networks contain many entities that interact in many levels and scales. According to Alexander, they must be treated as a system rather than separate objects that act within the urban context.

When looking into cities, it always comes down to urban agents that interact with each other. These agents can be individual human beings, families, households, organizations, municipalities, firms, and so on. They interact in a variety of domains such as economic, infrastructures, transportation facilities, commuting, internal immigration and more.

Since the works of Watts and Strogatz [1] and of Barabasi and Albert [2], the science of complex networks has been developing rapidly. It has been applied to various disciplines in order to study the topology of large networks and to understand their development and robustness [3-11]. Most of these works, however, consider the topological characteristics of urban networks, while the physical aspect is mostly neglected.

Cities are typical examples of complex, self-organizing systems [10-12]. They have originally emerged and are still developing out of the interactions between many agents that are located and move in space and time $[13,14]$. These agents are motivated by a variety of forces ranging from cognitive capabilities and needs to economic considerations, political ambitions, etc., with no central force that affects their behavior. These interactions entail a huge number of links that create complex networks which form the city.

Most if not all complexity theories and models have been applied to the study of cities with the implication that we now have a whole family of urban simulation models that model cities (See review in $[11,15])$. In the last decade or so cellular automata and agentbase models $(\mathrm{CA} / \mathrm{AB})$ have become the main medium to simulate cities as complex systems [15-17] while in the last few years we see studies that model cities as complex networks that are often typified by power law distributions [12, 18-19].

In this work, we focus on urban networks and study their topological as well as spatial characteristics. We aim to identify a set of fundamental rules that govern the interactions within urban systems at the metropolitan scale. For that, we developed a USM (Urban Simulation Model) specifically designed to study the evolution and dynamics of systems of cities. Our model is innovative in its structure as it is a superposition of cellular automata and agent based modeling approaches (that are essentially spatial analyses) and a complex network approach (that is essentially a topological analysis). This implies that in our model, the local activities and interaction of agents give rise to the global urban structure and network that in turn affects the agents' cognition, behavior, movement and action in the city and so on in circular causality.

In the next section we present the detailed description of the above model. Then, we present some preliminary results. As the presented work is an ongoing one, in the last section, we elaborate on directions for future work.

\section{A Detailed Description of the Model}

The essence of our model can be described by the following scenario: we start with a metropolitan environment divided into sub-areas, some of which represent cities with 
their municipal boundaries and others represent green areas. Each city is growing logistically (e.g. demographically due to natural increase and/or migration), and economically due to the development of employment and business centers. The residents (agents) of the city seek working places either in their town of residence or in other cities (to which they need to commute). Their decision where to work in based on a gravitation spatial interaction model i.e. their choice of a preferred working place is proportional to the size of the existing employment centers and inversely proportional to the distance between the agent's home and employment center. The choices where to work give rise to an intra- and inter-city commuting system. When a certain threshold of commuters between two cities is crossed, a link between these cities is created. The weight of the link is a dynamic parameter, which represents the volume of commuters.

Our USM is composed of the following elements and process:

1. The metropolis infrastructure:

(a) The area of the metropolis which is represented by a rectangular is defined (by the user)

(b) The model divides the metropolis into 25 spatial equal units and characterizes 7 of them (based on a random choice) as green areas (where urban development is restricted)

(c) The remaining 18 urban units are randomly united into 12 municipalities. These numbers are comparable to the Tel Aviv metropolis (to which we intend to relate this work in later stages).

(d) For each municipality, a center is randomly selected and being occupied by one of the agents. This random choice of the urban center can be explained as a historical accident [12].

2. Characterizing up to 3 types of agents. Each type of agents is represented by:

(a) Growth rate - the addition of new agents in each iteration

(b) Sensitivity to size of employment opportunities ( $\boldsymbol{\alpha}$ - see below)

(c) Sensitivity to distance between the agent's place of residence and work ( $\boldsymbol{\beta}$ - see below)

3. Location method:

(a) At the first stage: for each municipality an agent is randomly selected and located. Each new agent can be located either in its original city or elsewhere based on the availability of vacant space and a probability function. If the agent is not located in its original town its new location is determined by a gravitation function. The gravitation function can be described by: $G=\frac{(M m)^{\alpha}}{r^{\beta}}$, where $\boldsymbol{G}$ represents the gravitation force, $\boldsymbol{M}$ and $\boldsymbol{m}$ represent the size (total number of agents) in the agent's original and destination towns (correspondingly), $r$ represents the physical distance between the centers of both towns, and $\boldsymbol{\alpha}$ and $\boldsymbol{\beta}$ are parameters that characterize the sensitivity of the agent to size and distance correspondingly.

(b) The segregation function can be turned On/Off. This determines whether agents want to locate close to agent of their type.

4. Network definition:

(a) Nodes are defined at the location of the first agent in each city. The historical center is considered as the city's core 
(b) A minimum volume of commuters (agents) is needed to define a link. This number is predefined as a percentage of the population (and is changeable)

(c) The weight of the links is presented (gradient color change)

(d) When a predefined percentage of the cells, located in proximity to the municipal boundaries are occupied by agents, an agglomeration emerges. This means that the municipal boundaries are ignored in the gravitation function and the new center of the agglomeration is moved to its geographical center. Note that this condition is ignored when the predefined percentage of cells, located near the municipal boundary is 100 .

\section{Preliminary Results}

Based on the above USM, we can introduce some interesting preliminary results. In figures 1-2 (bottom), the green rectangles represent green areas where urban development is restricted, while the black lines represent municipal boundaries. The different types of agents are represented by black, red, and grey pixels. The first issue we address concerns agglomerations in face of municipal boundaries. Figure 1 presents the morphology and the network topology of the metropolis in two different runs of the model. In both runs there is only one type of agents. In the first run of the model (figure 1a), the agents consider the municipal boundaries in their choice of working places, while in the second run (figure $1 \mathrm{~b}$ ) the agents consider agglomerations instead of municipalities in their choice of working places. It can be seen that the perception of urban boundaries plays a significant role in the metropolitan evolution in terms of network topology. While in figure 1a (top) the topology of the network is a star network, in figure $1 \mathrm{~b}$ (top) the star network disappears and a new topology emerges. This new topology suggests that the existence of business centers, belonging to different municipalities but located in proximity to each other, might lead to the emergence of new centers at the metropolitan scale. In other words, employment centers at the metropolitan scale can span beyond the boundaries of a single municipality.

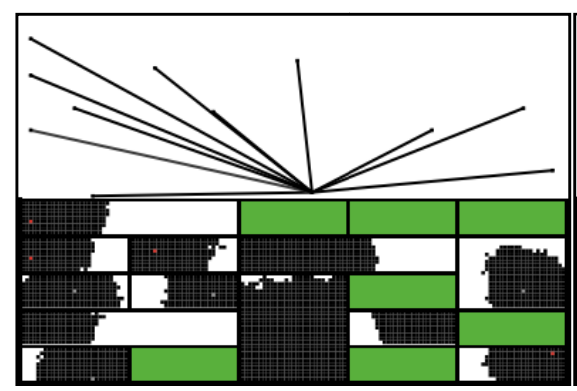

(a)

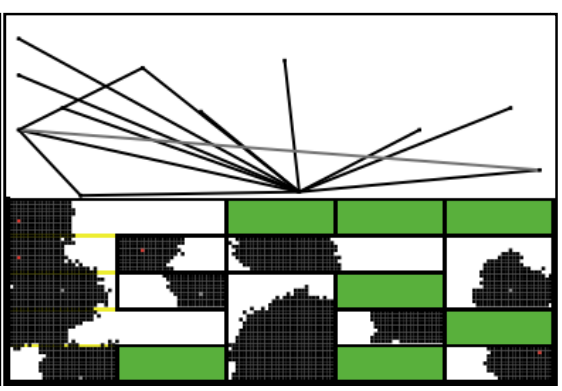

(b)

Fig. 1. The model at the metropolitan scale with one type of agents: a) municipal boundaries are considered and b) agglomerations of built-up areas replace municipalities 
The second issue concerns urban residential segregation. Figure 2 presents the morphology and network topology of the metropolitan in two different runs of the model in which there are 3 different types of agents. In the first run the agents are indifferent to the kind of their neighbors, thus, they might be located in adjacency to other kinds of agents (figure $2 \mathrm{a}$, bottom). In the second run the agents prefer to locate in proximity to agents of their own kind (figure $2 \mathrm{~b}$ bottom). To control this behavior, we added a segregation function to the model. When in use, this function affects the gravitation function such that $\mathrm{M}$ and $\mathrm{m}$ are calculated based only on the number of agents of the same kind as the agent who looks for a location.

It can be seen that the location preferences of the agents regarding proximity to similar/different agents have a major affect both on the urban morphology and network topology. When the agents prefer to locate in proximity to their own kind - urban segregation emerges. In terms of network topology, a significant difference between both runs is observed. When plotting the rank-size distribution of the links' weight ( $Y=\log ($ weight of link) and $\mathrm{X}=\log ($ rank of link)) the distribution of the first run (agents have no preferences of neighbors) corresponds to an exponential function (figure $3 \mathrm{a}$ ), while the distribution of the second run (agents prefer to work in proximity to their own kind) obeys a power law (figure $3 b$ ).

In addition, in the first run the highest level of commuters reaches approximately $11 \%$ of the total population in the metropolis. On the other hand, in the second run the highest level of commuters reaches only $6.7 \%$ of the total population. This can be explained by the fact that in the first run, the large employment centers attracted all three types of agents. However, in the second run, employment centers attracted only agents of the same type as their majority population.

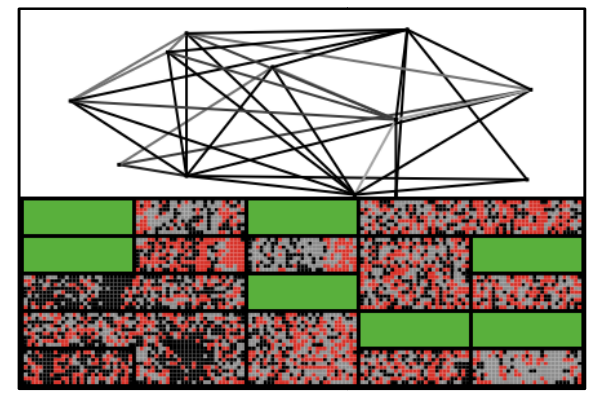

(a)

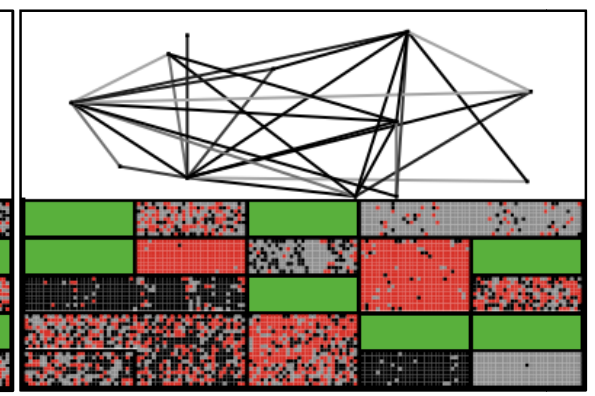

(b)

Fig. 2. The model at the metropolitan scale with 3 types of agents: a) agents are tolerant to location in proximity to other types of agents b) agents prefer to locate in proximity to their own types 

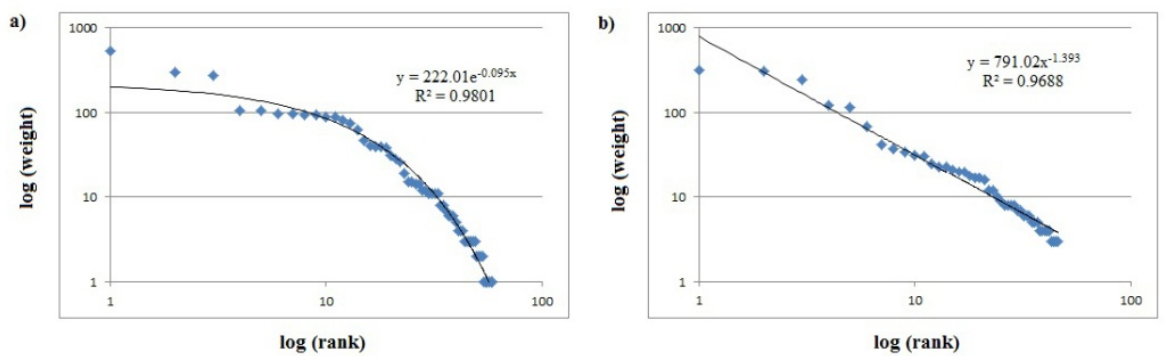

Fig. 3. Rank size distribution of links' weight: a) when agents are tolerant to location in proximity to other types of agents and b) when agents prefer to locate in proximity to their own types

Lastly, we address the rank-size distribution of the agents in our model. There is an extensive work on scaling relationship in cities [20-25]. One of the classic works is that of Haggett [26] who suggested that city size distributions of urban systems can be divided into three classes: the first includes system of cities with primate city (the largest city is considerably larger than the next largest one), the second class includes power low distributions and the third includes systems of cities in which the large cities are rather homogenously distributed. An empirical study on the size distribution of cities [25] showed that homogenous systems of cities can indeed be classified into these classes. When analyzing the resulted size distributions of the different agents in our model, we get all the observed population size distributions (see examples in figure 4). This, of course corresponds to the different values of parameters we used in different runs. This is also valid to the network topology. We found that the rank size distributions of the links' weight also correspond to these three classes. These results suggest that the set of laws to which the agents in our model obey, correspond to the behavior of people in real systems of cities. By following these laws and the values of the parameters that represent them we can try and understand which forces are the most dominant in the creation of scaling relationship within urban systems (as opposed to other parameters that lead to other relations).

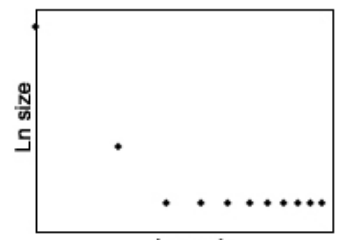

Ln rank

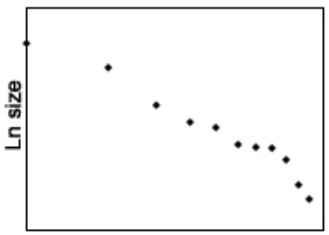

Ln rank

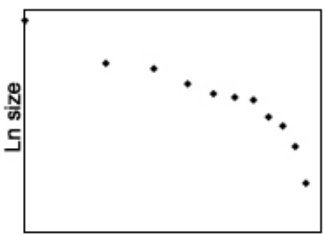

Ln rank

Fig. 4. The three classes of city size distributions, recovered by different runs of our model (with different parameters at each run) 


\section{Discussion and Conclusion}

In this paper we've presented an urban simulation model designed to study the evolution and dynamics of metropolitan systems of cities. Our model is built as a superposition of cellular automata and agent based modeling approaches (that are essentially spatial analyses) and a complex network approach (that is essentially a topological analysis). We study both the topology and the spatial characteristics of urban commuting networks at the metropolis scale.

In a future work, we intend to elaborate our model in order to study additional urban phenomena; the first addresses the morphology of the municipal boundaries. To explore this issue we will change the definition of municipal boundaries and study the effect of increased irregularity of the boundaries' morphology on the resulted networks. The second phenomenon addresses preservation of rural environment under the economic pressures of real-estates developers. In reality rural areas are often transformed into developed urban environments (e.g. edge cities). In our next version of the model, we will enable (under controlled conditions) urban development in the rural (green) areas and study its effect on the urban networks in terms of the networks' topology and spatial characteristics.

\section{References}

1. Watts, D.J., Strogatz, S.H.: Collective dynamics of 'small-world' networks. Nature 393(6684), 409-410 (1998)

2. Barabási, A.L., Albert, R.: Emergence of Scaling in Random Networks. Science 286(5439), 509-512 (1999)

3. Albert, R., Jeong, H., Barabási, A.L.: Error and attack tolerance of complex networks. Nature 406, 378-382 (2000)

4. Gallos, L.K., Cohen, R., Argyrakis, P., Bunde, A., Havlin, S.: Stability and topology of scale-free networks under attack and defense strategies. Phys. Rev. Lett. 94, 188701 (2005)

5. Paul, G., Sreenivasan, S., Havlin, S., Stanley, H.E.: Optimization of network robustness to random breakdowns. Physica A 370, 854-862 (2006)

6. Jeong, H., Tombora, B., Albert, R., Oltvai, Z.N., Barabási, A.L.: The large-scale organization of metabolic networks. Nature 407, 651-654 (2000)

7. Newman, M., Watts, D.J., Strogatz, S.H.: Random graph models of social networks. PNAS 99, 2566-2572 (2002)

8. Jeong, H., Neda, Z., Barabási, A.L.: Measuring preferential attachment in evolving networks. Europhys. Lett. 61, 567-572 (2003)

9. Blumenfeld-Lieberthal, E.: The topology of transportation networks: a comparison between different economies. Networks and Spatial Economics 9, 427-458 (2009)

10. Patuelli, R., Reggiani, A., Nijkamp, P., Bade, F.J.: The evolution of the commuting network in Germany: spatial and connectivity patterns. Journal of Transport and Land Use 2(3) (2010)

11. Batty, M.: Cities and complexity. The MIT Press, Cambridge (2005)

12. Porutgali, J.: Complexity, Cognition and the City. Springer, Heidelberg (2011)

13. Portugali, J.: Self-Organization and the City. Springer, Berlin (2000) 
14. Alexander, C.: A city is not a tree. Architectural Forum 122(1), 58-61 and 122(2), 58-62 (1965)

15. Batty, M., Longley, P.: Fractal Cities: A Geometry of Form and Function. Academic Press, London (1994)

16. Benenson, I., Torrens, P.: Geosimulation: Automata-based modeling of urban phenomena. John Wiley and Sons Ltd., West Sussex (2004)

17. White, R., Engelen, G.: Cellular automata and fractal urban form: a cellular modeling approach to the evolution of urban land-use patterns. Environment and Planning A 25, 1175-1199 (1993)

18. Andersson, C., Frenken, K., Hellervik, A.: A complex network approach to urban growth. Environment and Planning A 38, 1941-1964 (2006)

19. Porta, S., Crucittib, P., Latora, V.: The network analysis of urban streets: A dual approach. Physica A 369, 853-866 (2006)

20. Jiang, B., Claramunt, C.: Topological analysis of urban street networks. Environment and Planning B 31(1), 151-162 (2004)

21. Zipf, G.K.: National unity and disunity. The Principia Press, Bloomington Indiana (1941)

22. Krugman, P.: The Self-Organizing Economy. Blackwell, Cambridge (1996)

23. Batty, M.: Rank clocks. Nature 444, 592-596 (2006)

24. Geoffery, F., Linsker, R.: Synchronous neural activity in scale-free network models versus random network models. PNAS 102(28), 9948-9953 (2005)

25. Benguigui, L., Blumenfeld-Lieberthal, E.: A new classification of city size distributions. Computers, Environment and Urban Systems 31(6), 648-666 (2006)

26. Haggett, P.: Models in geography: the Madingley lectures for 1965 . Edited with Chorley, R.J., Methuen \& Co. Ltd., London (1967) 